\title{
Three-To-One Internal Resonance of Inclined Marine Riser
}

\author{
Feras K. Alfosail Mohammad I. Younis \\ Physical Sciences and Engineering Division, King Abdullah University of Science \\ and Technology, Thuwal 23955-9600, Saudi Arabia.
}

\begin{abstract}
In this paper, we investigate the three-to-one internal resonance of an inclined marine riser. The model accounts for the flexural rigidity of the riser, variable axial load, nonlinear geometry, and initial static deflection due to self-weight. The applied tension with the static deflection are tuned such that the ratio between the fifth and first natural frequencies is three. Then, the multiple time scales (MTS) perturbation method is applied to study the internal resonance interactions when the structure is harmonically excited. The system equation is solved using a multi-mode Galerkin model and its results are compared to the perturbation results showing good agreement. Moreover, the frequency response curves of the fifth and first modes amplitudes exhibit Hopf and saddle node bifurcations. In addition, the interaction of the fifth mode with the first mode during internal resonance results into new emerging solutions and states. These phenomena are well observed in the force response curves and confirmed by the time history of the response of the structure, which can lead to complex dynamics that hinder the life of the riser by fatigue failure.
\end{abstract}

Keywords: nonlinear oscillations, marine riser, three-to-one internal resonance, vortex induced vibrations.

*Corresponding Author:

Mohammad I. Younis, e-mail: mohammad.younis@kaust.edu.sa, tel.:+966544700154 


\section{Introduction}

Nonlinear dynamics of marine risers have attracted considerable research over the past decades. The riser structure, under the influence of environmental factors and geometric nonlinearities, can exhibit complex vibration phenomena, such as heave motions and vortex induced vibrations (VIV). These dynamical features are of interest to understand how the riser structure behave, prevent its failure by fatigue [1], and utilize the motion of the structure, for instance, in energy harvesting applications [2]. In addition, the activation of veering [3] and internal resonance [4] in the response of the riser structure leads to an increase in the complexity of the dynamics that require more thorough investigation.

The modal interaction via internal, autoparametric, resonance, involves the transfer and exchange of energy from one mode to another upon exciting the nonlinear system. Internal resonances have been investigated in cables, beams, and plates more information can be found in [5]. Among these structures, the most commonly studied internal resonances are one-to-one $(1: 1)$, two-to-one $(2: 1)$, and three-to-one $(3: 1)$. In this work, we investigate the three-to-one internal resonance because there is a possibility that it gets excited under environmental loads causing higher order frequency components of the system to exist; which accelerate fatigue failure of the riser.

The analysis of marine riser structure exhibits certain similarity to those of classic beams. The variable axial force in the riser equation is often simplified by its average applied load reducing the equation to a beam equation [6]. Many researchers have investigated the three-toone internal resonance in beams. Earlier work in this field is attributed to Nayfeh et al. [7] who studied the three-to-one internal resonance between the first and second modes of a hinged- 
clamped beam constrained with a spring at the clamped boundary. They revealed that the system exhibit features that can lead to Hopf bifurcations. Chin and Nayfeh [8] extended the work to study the bifurcations and examined the dynamical solutions of the system. They showed using the method of normal forms that the type of Hopf bifurcation depends on the axial load and the system after Hopf bifurcation undergoes multiple period doubling bifurcations and series of jump responses. Emam and Nayfeh [9] studied the nonlinear response of a buckled beam under a three-to-one internal resonance between the first and the third mode. They used multiple scales to examine the time response and study the stability of the vibration of the beam around the buckled configurations. Additionally, Wang et al. [10] investigated the influence of elastic foundation on the response of the beam while considering three-to-one internal resonance.

Marine risers are also modeled using nonlinear Euler-Bernoulli beam theories accounting for the variable applied axial load due to self-weight. When the riser top is at an offset from the seabed, the self-weight induces a distributed load that causes a static deflection of the structure introducing new linear and nonlinear terms. Although, an analytical solution to the static problem exists in the form of a catenary solution [11] or that of an inclined beam [12], there is no analytical solution for the dynamic problem, while considering the static deflection and the variable axial load, and particularly accounting for the internal resonance. Therefore, numerical studies have been presented recently to address the nonlinear interactions in marine risers. Examples of these studies include the work of Mazzilli et al. $[13,14]$ who developed a finite element invariant manifold procedure for vertical and catenary risers that takes into account the influence of internal resonance. Alternatively, cable models have been employed while accounting for the internal resonance $[15,16]$. Extension of these models by considering bending is realized in the work of Srinil et al. $[17,18]$ who studied the vortex induced vibration of the 
riser considering the two-to-one internal resonance. Other works in the field considered the parametric resonance of risers combined with internal resonance, such as the work of Chatjigeorgiou and Mavrakos [19] and Tang et al. [20].

In an earlier study [21], we have demonstrated that tuning the offset and the applied top tension introduce potentially several internal resonances among the various vibration modes, such as two-to-one, three-to-one, and four-to-one. Due to the lack of extensive research on this subject, the aim of this paper is to investigate the three-to-one internal resonance of the riser accounting for the static deflection and mid-plane stretching while the riser is subjected to harmonic excitation. The organization of this paper is as follows: The problem formulation is discussed in Section 2. Perturbation analysis of the equation of motion is discussed in Section 3. Section 4 addresses the validation and discussion of the results obtained by perturbation. Finally, the concluding remarks are given in Section 5.

\section{Problem Formulation}

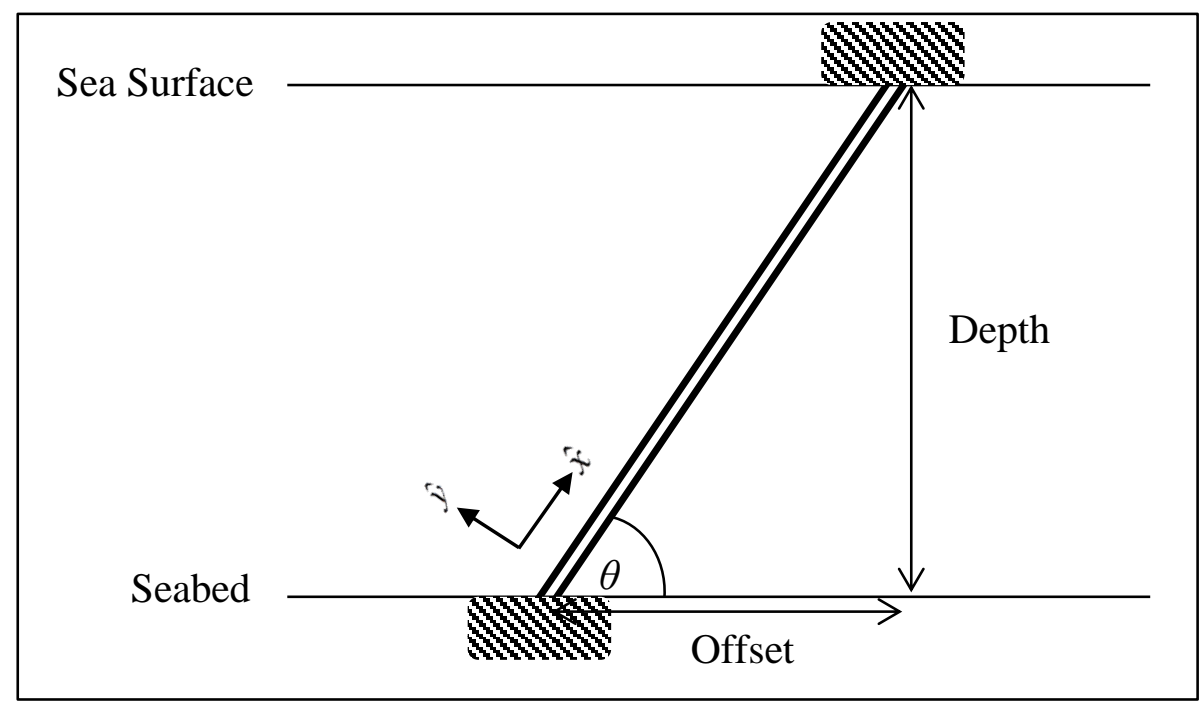

Figure 1. Schematic of the inclined riser used in the analysis. 
We consider analyzing the inclined riser shown in Fig. 1. The riser is fixed at both ends and extends from the sea surface to the sea bed. The depth and offset are used as geometric parameters to characterize the inclination angle of the riser. The sea current direction is assumed to be orthogonal to the plane of curvature of the riser. Then, the equation of motion of the riser is described by [22]

$$
\begin{aligned}
& m \frac{\partial^{2} \hat{y}_{t}}{\partial \hat{t}^{2}}+E I \frac{\partial^{4} \hat{y}_{t}}{\partial \hat{x}^{4}}-\left(T_{e}-W_{e} \sin (\theta)(L-\hat{x})+\frac{E A_{p}}{2 L} \int_{0}^{L}\left[\left(\frac{\partial \hat{y}_{t}}{\partial \hat{x}}\right)^{2}\right] d \hat{x}\right)\left(\frac{\partial^{2} \hat{y}_{t}}{\partial \hat{x}^{2}}\right)-W_{e} \sin (\theta)\left(\frac{\partial \hat{y}_{t}}{\partial \hat{x}}\right) \\
& +\bar{c} \frac{\partial \hat{y}_{t}}{\partial \hat{t}}=F_{d}-W_{e} \cos (\theta)
\end{aligned}
$$

subjected to the boundary conditions

$\hat{y}_{t}(\hat{x}, \hat{t})=0$ at $\hat{x}=0$ and $\hat{x}=L$

$\frac{\partial \hat{y}_{t}}{\partial \hat{x}}$ at $\hat{x}=0$ and $\hat{x}=L$

where $\hat{y}_{t}$ is the deflection of the riser along position $\hat{x}$ and time $\hat{t}, m$ is the total mass per unit length, which includes the added mass per unit length $m_{a}$ and the mass of the riser pipe $m_{p}, E I$ is flexural rigidity, $W_{e}$ is the apparent weight of the riser per unit length defined as $W_{e}=\left(m_{p}-\rho_{e} A_{e}\right) g, \rho_{e}$ is the density of seawater, $A_{e}$ is the area based on the outside diameter of the riser pipe, $T_{e}$ is the applied tension, $\theta$ is the inclination angle, $L$ is the length of the pipe, $A_{p}$ is the cross section area of the pipe, $\bar{c}$ is the damping coefficient and $F_{d}$ is the external dynamic forces. The axial tension of the pipe is assumed to vary linearly with the length of the pipe. To obtain the static and dynamic parts of the riser structure we assume the total riser deflection 
$\hat{y}_{t}(\hat{x}, \hat{t})$ to be a superposition of a static component $\hat{y}_{s}(\hat{x})$ and a dynamics component $\hat{y}_{d}(\hat{x}, \hat{t})$ as follows

$$
\hat{y}_{t}(\hat{x}, \hat{t})=\hat{y}_{s}(\hat{x})+\hat{y}_{d}(\hat{x}, \hat{t})
$$

The static differential equation of the inclined riser is obtained by dropping the time dependent terms in Eq. (1.1), which results into

$$
E I \frac{d^{4} \hat{y}_{s}}{d \hat{x}^{4}}-\left(T_{e}-W_{e} \sin (\theta)(L-\hat{x})+\frac{E A_{p}}{2 L} \int_{0}^{L}\left[\left(\frac{d \hat{y}_{s}}{d \hat{x}}\right)^{2}\right] d \hat{x}\right)\left(\frac{d^{2} \hat{y}_{s}}{d \hat{x}^{2}}\right)-W_{e} \sin (\theta)\left(\frac{d \hat{y}_{s}}{d \hat{x}}\right)=-W_{e} \cos (\theta)
$$

Equation (3) is solved using the boundary layer perturbation technique, the method of matched asymptotic expansion [23], considering the tension is dominant over bending and other contributions. The dynamic equation of motion of the inclined riser is obtained by dropping the terms of the static Eq. (3), after substituting Eq. (2) into Eq. (1). Then, the resulting dynamic equation is written as

$$
\begin{aligned}
& m \frac{\partial^{2} \hat{y}_{d}}{\partial \hat{t}^{2}}+E I \frac{\partial^{4} \hat{y}_{d}}{\partial \hat{x}^{4}}-\left(T_{e}-W_{e} \sin (\theta)(L-\hat{x})\right)\left(\frac{\partial^{2} \hat{y}_{d}}{\partial \hat{x}^{2}}\right)-W_{e} \sin (\theta)\left(\frac{\partial \hat{y}_{d}}{\partial \hat{x}}\right)+\bar{c} \frac{\partial \hat{y}_{d}}{\partial \hat{t}} \\
& -\frac{E A_{p}}{2 L}\left(\int_{0}^{L}\left[2 \frac{d \hat{y}_{s}}{d \hat{x}} \frac{\partial \hat{y}_{d}}{\partial \hat{x}}\right] d \hat{x}+\int_{0}^{L}\left[\left(\frac{\partial \hat{y}_{d}}{\partial \hat{x}}\right)^{2}\right] d \hat{x}\right)\left(\frac{d^{2} \hat{y}_{s}}{d \hat{x}^{2}}\right) \\
& -\frac{E A_{p}}{2 L}\left(\int_{0}^{L}\left[\left(\frac{d \hat{y}_{s}}{d \hat{x}}\right)^{2}\right] d \hat{x}+\int_{0}^{L}\left[\left(\frac{\partial \hat{y}_{d}}{\partial \hat{x}}\right)^{2}\right] d \hat{x}+\int_{0}^{L}\left[2 \frac{d \hat{y}_{s}}{d \hat{x}} \frac{\partial \hat{y}_{d}}{\partial \hat{x}}\right] d \hat{x}\right)\left(\frac{\partial^{2} \hat{y}_{d}}{\partial \hat{x}^{2}}\right)=F_{d}
\end{aligned}
$$

To simplify the analysis, we introduce the following dimensionless variables

$$
y=\frac{\hat{y}}{D} \quad x=\frac{\hat{x}}{L} \quad t=\sqrt{\frac{E I}{m L^{4}}} \hat{t}
$$

where $D$ is the outer diameter of the riser pipe. The "hat" denotes the dimensional variables while the variables without "hat" are dimensionless. Substituting Eq. (5) into (4) yields 


$$
\begin{aligned}
& \frac{\partial^{2} y_{d}}{\partial t^{2}}+\frac{\partial^{4} y_{d}}{\partial x^{4}}-\left(\bar{T}-\bar{\sigma}(1-x)-\bar{\eta}\left(\int_{0}^{1}\left(\frac{d y_{s}}{d x}\right)^{2} d x+\int_{0}^{1}\left(\frac{\partial y_{d}}{\partial x}\right)^{2} d \hat{x}+\int_{0}^{1} 2 \frac{d y_{s}}{d x} \frac{\partial y_{d}}{\partial x} d \hat{x}\right)\right)\left(\frac{\partial^{2} y_{d}}{\partial x^{2}}\right) \\
& -\left(\bar{\eta}\left(\int_{0}^{1} 2 \frac{d y_{s}}{d x} \frac{\partial y_{d}}{\partial x} d x+\int_{0}^{1}\left(\frac{\partial y_{d}}{\partial x}\right)^{2} d \hat{x}\right)\right)\left(\frac{d^{2} y_{s}}{d x^{2}}\right)-\bar{\sigma}\left(\frac{\partial y_{d}}{\partial x}\right)+c \frac{\partial y_{d}}{\partial t}=\bar{F}_{d}
\end{aligned}
$$

where

$$
\bar{\sigma}=\frac{W_{e} \sin (\theta) L^{3}}{E I} ; \quad \bar{\eta}=\frac{A_{p} D^{2}}{2 I} ; \quad c=\frac{\bar{c} L^{2}}{\sqrt{m E I}} ; \quad \bar{F}_{d}=\frac{L^{4} F_{d}}{E I D} ; \quad \bar{T}=\frac{T_{e} L^{2}}{E I}
$$

The boundary conditions become

$y(x, t)=0$ at $x=0$ and $x=1$

$\frac{\partial y}{\partial \hat{x}}=0$ at $x=0$ and $x=1$

The dynamic equation has a cubic nonlinearity introduced by the mid-plane stretching. In addition, the static solution from Eq. (3) introduces quadratic nonlinearities. The next step is to study the linear eigenvalue problem to obtain the natural frequencies and mode shapes. Equations (6) is linearized by dropping damping, forcing, and the nonlinear terms yielding

$$
\frac{\partial^{2} y_{d}}{\partial t^{2}}+\frac{\partial^{4} y_{d}}{\partial x^{4}}-\left(\bar{T}+\bar{\sigma} x+\bar{\eta} \int_{0}^{1}\left(\frac{d y_{s}}{d x}\right)^{2} d x\right) \frac{\partial^{2} y_{d}}{\partial x^{2}}-\bar{\eta}\left(\int_{0}^{1} 2 \frac{d y_{s}}{d x} \frac{\partial y_{d}}{\partial x} d x\right)\left(\frac{d^{2} y_{s}}{d x^{2}}\right)-\bar{\sigma} \frac{\partial y_{d}}{\partial x}=0
$$

where $\bar{T}_{s}=\bar{T}-\bar{\sigma}$. The mode shapes and the natural frequencies of the structure are extracted by solving Eq. (8), using unforced beam mode shapes, via the Galerkin method [21]. For the analysis of the forced vibration part, one needs to consider the forces induced by vortex induced vibrations because they are the most detrimental to the riser structure. Conventionally, computational fluid dynamics $[24,25]$ and semi-empirical formulations [26-28] are utilized to 
describe the fluid lift and drag forces on the riser structure; but the utilization of these methods increases the complexity when nonlinear analysis tools are used such as multiple scales method. Therefore, in this work, the vortex induced vibration force is described by a simple force model given by [29]

$\bar{F}_{d}=f \cos (\Omega t)$

where $f$ is defined as $f=\frac{1}{2} \frac{\rho_{e} U^{2} L^{4} C_{L}}{E I}, U$ is the sea current velocity, and $C_{L}$ is the average lift coefficient.

\section{Perturbation Analysis}

In this section, we use the method of multiple time scales MTS to analyze the nonlinear response of the riser subjected to three-to-one internal resonance. The MTS expansion is applied directly on the integral differential equation given by Eq. (6). Hence, we seek a third-order expansion of the form

$y_{d}(x, t ; \varepsilon)=\varepsilon y_{1}\left(x, T_{0}, T_{2}\right)+\varepsilon^{2} y_{2}\left(x, T_{0}, T_{2}\right)+\varepsilon^{3} y_{3}\left(x, T_{0}, T_{2}\right)$

where $\varepsilon$ is introduced as a bookkeeping parameter, $T_{0}=\varepsilon t$, and $T_{2}=\varepsilon^{3} t$. The auto scaling in Eq. (10) determines that the quadratic nonlinearities are at the second order and the cubic nonlinearities are at the third order. Furthermore, the scales of forcing and damping are assumed to be $\varepsilon^{3} f \cos (\Omega t)$ and $\varepsilon^{2} c$, respectively. Substituting Eq. (10) into Eq. (6) yields

Order $\varepsilon$ 
$\ell\left(y_{1}\right)=D_{0}^{2} y_{1}+y_{1}^{i v}+\bar{T}_{s} y_{1}^{\prime \prime}-x \bar{\sigma} y_{1}^{\prime \prime}-\bar{\sigma} y_{1}^{\prime}-2 \eta y_{s}^{\prime \prime}(x)\left(\int_{0}^{1} y_{s}^{\prime}(x) y_{1}^{\prime} \mathrm{d} x\right)=0$

Order $\varepsilon^{2}$

$$
\ell\left(y_{2}\right)=-2 D_{0} D_{1} y_{1}+\eta y_{s}^{\prime \prime}(x)\left(\int_{0}^{1}\left(y_{1}^{\prime}\right)^{2} \mathrm{~d} x\right)+2 \eta y_{1}^{\prime \prime}\left(\int_{0}^{1} y_{s}^{\prime}(x) y_{1}^{\prime} \mathrm{d} x\right)
$$

Order $\varepsilon^{3}$

$$
\begin{aligned}
\ell\left(y_{3}\right) & =-2 D_{0} D_{1} y_{2}-2 D_{0} D_{2} y_{1}-D_{1}^{2} y_{1}+\eta y_{1}^{\prime \prime}\left(\int_{0}^{1}\left(y_{1}^{\prime}\right)^{2} \mathrm{~d} x\right)+\frac{f}{2} e^{\mathrm{i} \Omega \mathrm{T}_{0}}+2 \eta y_{s}^{\prime \prime}\left(\int_{0}^{1} y_{1}^{\prime} y_{2}^{\prime} \mathrm{d} x\right) \\
& +2 \eta y_{2}^{\prime \prime}\left(\int_{0}^{1} y_{s}^{\prime}(x) y_{1}^{\prime} \mathrm{d} x\right)+2 \eta y_{1}^{\prime \prime}\left(\int_{0}^{1} y_{s}^{\prime}(x) y_{2}^{\prime} \mathrm{d} x\right)-c D_{0} y_{1}
\end{aligned}
$$

where the temporal derivatives are defined as $D_{0}^{2}=\frac{\partial}{\partial T_{0}^{2}}, D_{0}=\frac{\partial}{\partial T_{0}}, D_{1}^{2}=\frac{\partial}{\partial T_{1}^{2}}, D_{1}=\frac{\partial}{\partial T_{1}}$, $D_{2}=\frac{\partial}{\partial T_{2}},()^{\prime}$ denotes the derivative with respect to $x$, and $\ell($ ) defines the linear operator given by Eq. (11). It is observed that the expansion at the first order $\varepsilon$, Eq. (11), yields the linear eigenvalue problem defined in Eq. (8). Then, the presence of the internal resonance yields coupled modes interaction where the solution of Eq. (11) can be sought to be of the form

$y_{1}=A_{n}\left(T_{2}\right) \phi_{n}(x) e^{i \omega_{n} T_{0}}+A_{m}\left(T_{2}\right) \phi_{m}(x) e^{i \omega_{m} T_{0}}+\bar{A}_{n}\left(T_{2}\right) \phi_{n}(x) e^{-i \omega_{n} T_{0}}+\bar{A}_{m}\left(T_{2}\right) \phi_{m}(x) e^{-i \omega_{m} T_{0}}$

where $A_{n}$ and $A_{m}$ are the amplitudes corresponding to modes $m$ and $n, \phi_{n}(x)$ and $\phi_{m}(x)$ are the $m^{\text {th }}$ and $n^{\text {th }}$ mode shapes while $\omega_{n}$ and $\omega_{m}$ are the corresponding natural frequencies. Since the activation of the three-to-one internal resonance occurs at the third order, we impose to have the 
amplitudes $A_{n}$ and $A_{m}$ function of $T_{2}$ only where the solvability condition is obtained. Then, Eq. (14) is substituted in the second order Eq. (12) to obtain

$$
\begin{aligned}
\ell\left(y_{2}\right)= & \left(\eta y_{s}^{\prime \prime}\left(\int_{0}^{1}\left(\phi_{m}^{\prime}\right)^{2} \mathrm{~d} x\right)+2 \eta \phi_{m}^{\prime \prime}\left(\int_{0}^{1} y_{s}^{\prime} \phi_{m}{ }^{\prime} \mathrm{d} x\right)\right)\left(A_{m}^{2} e^{2 i \omega_{m} T_{0}}+2 A_{m} \bar{A}_{m}\right) \\
& +\left(\eta y_{s}^{\prime \prime}\left(\int_{0}^{1}\left(\phi_{n}^{\prime}\right)^{2} \mathrm{~d} x\right)+2 \eta \phi_{n}^{\prime \prime}\left(\int_{0}^{1} y_{s}^{\prime} \phi_{n}^{\prime} \mathrm{d} x\right)\right)\left(A_{n}^{2} e^{2 i \omega_{n} T_{0}}+2 A_{n} \bar{A}_{n}\right) \\
& +\Lambda_{n m}\left(A_{n} \bar{A}_{m} e^{i\left(\omega_{n}-\omega_{m}\right) T_{0}}+A_{m} A_{n} e^{i\left(\omega_{m}+\omega_{n}\right) T_{0}}\right)+\mathrm{cc}
\end{aligned}
$$

where cc is the complex conjugate of the expression and $\Lambda_{n m}$ is defined by

$$
\Lambda_{n m}=2\left(\eta \phi_{m}^{\prime \prime} \int_{1}^{0} y_{s}^{\prime} \phi_{n}^{\prime} \mathrm{d} x+\eta \phi_{n}^{\prime \prime} \int_{1}^{0} y_{s}^{\prime} \phi_{m}^{\prime} \mathrm{d} x+\eta y_{s}^{\prime \prime} \int_{1}^{0} \phi_{m}^{\prime} \phi_{n}^{\prime} \mathrm{d} x\right)
$$

Because Eq. (15) has no secular terms, we consider the particular solution of Eq. (15), which has the following form:

$$
\begin{aligned}
y_{2}= & \Pi_{1 m}(x) A_{m}{ }^{2} e^{2 i \omega_{m} T_{0}}+\Pi_{2 m}(x) A_{m} \bar{A}_{m}+\Pi_{1 n}(x) A_{n}{ }^{2} e^{2 \omega_{n} T_{0}}+\Pi_{2 n}(x) A_{n} \bar{A}_{n} \\
& +\Pi_{1 m n}(x) A_{n} \bar{A}_{m} e^{i\left(\omega_{n}-\omega_{m}\right) T_{0}}+\Pi_{2 m n}(x) A_{m} A_{n} e^{i\left(\omega_{m}+\omega_{n}\right) T_{0}}+\mathrm{cc}
\end{aligned}
$$

where $\Pi_{1 m}, \Pi_{2 m}, \Pi_{1 n}, \Pi_{2 n}, \Pi_{1 m n}$ and $\Pi_{2 m n}$ are the solutions to the following boundary value problems defined by

$$
\begin{aligned}
& \mathrm{M}\left[\Pi_{1 i}(x) ; 2 \omega_{i}\right]=\eta y_{s}^{\prime \prime}\left(\int_{0}^{1} \phi_{i}^{\prime 2} \mathrm{~d} x\right)+2 \eta \phi_{i}^{\prime \prime}\left(\int_{0}^{1} y_{s}^{\prime} \phi_{i}^{\prime} \mathrm{d} x\right) \\
& \mathrm{M}\left[\Pi_{2 i}(x) ; 0\right]=2 \eta y_{s}^{\prime \prime}\left(\int_{0}^{1} \phi_{i}^{\prime 2} \mathrm{~d} x\right)+4 \eta \phi_{i}^{\prime \prime}\left(\int_{0}^{1} y_{s}^{\prime} \phi_{i}^{\prime} \mathrm{d} x\right)
\end{aligned}
$$

$\mathrm{M}\left[\Pi_{1 m n}(x) ; \omega_{n}-\omega_{m}\right]=\Lambda_{n m}$ 
$\mathrm{M}\left[\Pi_{2 m n}(x) ; \omega_{n}+\omega_{m}\right]=\Lambda_{n m}$

$\mathrm{M}[\Pi(x) ; \omega]$ is defined by

$\mathrm{M}[\Pi(x) ; \omega]=\Pi^{i v}-\omega^{2} \Pi-\bar{T}_{s} \Pi^{\prime \prime}-x \bar{\sigma} \Pi^{\prime \prime}-\bar{\sigma} \Pi^{\prime}-2 \eta y_{s}^{\prime \prime}\left(\int_{0}^{1} y_{s}^{\prime} \Pi^{\prime} \mathrm{d} x\right)$

After solving for the particular solution, we substitute solutions from Eq. (14) and Eq. (17) into the third order Eq. (13) reducing to

$$
\begin{aligned}
\ell\left(y_{3}\right)= & {\left[-2 \mathrm{i} \omega_{m} \phi_{m} D_{2} A_{m}-\mathrm{i} \omega_{m} c \phi_{m} A_{m}+\psi_{1}(x) \bar{A}_{m} A_{m}{ }^{2}+\psi_{2}(x) A_{n} \bar{A}_{n} A_{m}+\frac{f}{2} e^{i \alpha T_{2}}\right] e^{i \omega_{m} T_{0}} } \\
& +\left[-2 \mathrm{i} \omega_{n} \phi_{n} D_{2} A_{n}-\mathrm{i} \omega_{n} c \phi_{n} A_{n}+\psi_{3}(x) \bar{A}_{n} A_{n}{ }^{2}+\psi_{4}(x) A_{m} \bar{A}_{m} A_{n}\right] e^{i \omega_{n} T_{0}} \\
& +\left[\psi_{5}(x) A_{n} \bar{A}_{m}{ }^{2}\right] e^{i\left(\omega_{n}-2 \omega_{m}\right) T_{0}}+\left[\psi_{6}(x) A_{m}{ }^{3}\right] e^{3 i \omega_{m} T_{0}}+\mathrm{cc}+\mathrm{NST}
\end{aligned}
$$

where NST denotes non-secular terms, and $\psi_{i}(x)$ functions are defined in Appendix A. To obtain the solvability condition, the left hand side of Eq. (20) is multiplied with the adjoint of Eq. (14) $\phi_{j}(x) e^{ \pm \omega_{j} T_{0}}$. More details on the proof of self-adjointness are provided in Appendix B. Then, we consider the three-to-one internal resonance criterion given by $\omega_{n}=3 \omega_{m}+\delta \varepsilon^{3}$ around the first primary resonance $\Omega=\omega_{m}+\varepsilon^{3} \alpha$, where $\delta$ and $\alpha$ are detuning parameters, substitute in Eq. (20), and demand that the right hand side of the equation is orthogonal to $\phi_{m}(x) e^{-i \omega_{m} T_{0}}$ and $\phi_{n}(x)$ $e^{-i \omega_{n} T_{0}}$ to obtain the following solvability conditions:

$$
\begin{aligned}
& 2 \mathrm{i} \omega_{m} D_{2} A_{m}=-\mathrm{i} \omega_{m} 2 \mu_{1} A_{m}+8 R_{1} \bar{A}_{m} A_{m}{ }^{2}+8 R_{2} A_{n} \bar{A}_{n} A_{m}+8 R_{3} A_{n} \bar{A}_{m}^{2} e^{i \delta T_{2}}+F_{m} e^{i \alpha T_{2}} \\
& 2 \mathrm{i} \omega_{n} D_{2} A_{n}=-\mathrm{i} \omega_{n} 2 \mu_{2} A_{n}+8 K_{1} \bar{A}_{n} A_{n}^{2}+8 K_{2} A_{m} \bar{A}_{m} A_{n}+8 K_{3} A_{m}{ }^{3} e^{-i \delta T_{2}}
\end{aligned}
$$


where the coefficients $R_{i}, K_{i}, \mu_{1}, \mu_{2}$ are defined by

$$
\begin{aligned}
& 8 R_{1}=\int_{0}^{1} \phi_{m} \psi_{1} d x, \quad 8 R_{2}=\int_{0}^{1} \phi_{m} \psi_{2} d x, \quad 8 R_{3}=\int_{0}^{1} \phi_{m} \psi_{5} d x, 8 K_{1}=\int_{0}^{1} \phi_{n} \psi_{3} d x, \quad 8 K_{2}=\int_{0}^{1} \phi_{n} \psi_{4} d x, \\
& 8 K_{3}=\int_{0}^{1} \phi_{n} \psi_{6} d x, \quad 2 \mu_{1}=\int_{0}^{1} c \phi_{m}{ }^{2} d x, \quad 2 \mu_{2}=\int_{0}^{1} c \phi_{n}^{2} d x, \quad F_{m}=\frac{1}{2} \int_{0}^{1} f \phi_{m} \mathrm{~d} x
\end{aligned}
$$

Then, we use the polar transformation defined by

$$
\begin{aligned}
& A_{m}=\frac{1}{2} a_{m} e^{i \beta_{m}} \\
& A_{n}=\frac{1}{2} a_{n} e^{i \beta_{m}}
\end{aligned}
$$

Substituting Eq. (23.1) and Eq. (23.2) into Eq. (21.1) and Eq. (21.2) yields

$$
\begin{aligned}
& \omega_{m} D_{2} a_{m}=F_{m} \sin \left(\alpha T_{2}-\beta_{m}\right)+R_{3} a_{m}{ }^{2} a_{n} \sin \left(\beta_{n}-3 \beta_{m}+\delta T_{2}\right)-\mu_{1} \omega_{m} a_{m} \\
& \omega_{m} a_{m} D_{2} \beta_{m}=-F_{m} \cos \left(\alpha T_{2}-\beta_{m}\right)-R_{3} a_{m}{ }^{2} a_{n} \cos \left(\beta_{n}-3 \beta_{m}+\delta T_{2}\right)-R_{2} a_{m} a_{n}{ }^{2}-R_{1} a_{m}{ }^{3} \\
& \omega_{m} D_{2} a_{n}=-K_{3} a_{m}{ }^{3} \sin \left(\beta_{n}-3 \beta_{m}+\delta T_{2}\right)-\mu_{2} \omega_{n} a_{n} \\
& \omega_{m} a_{n} D_{2} \beta_{n}=-K_{3} a_{m}{ }^{3} \cos \left(\beta_{n}-3 \beta_{m}+\delta T_{2}\right)-K_{1} a_{m}{ }^{2} a_{n}-K_{1} a_{n}{ }^{3}
\end{aligned}
$$

Equations (24.1)-(24.4) represent a system of nonlinear ordinary differential equations that govern the phase and amplitude modulation. The equations are simplified further by introducing $\gamma_{1}=\alpha T_{2}-\beta_{m}$ and $\gamma_{2}=\beta_{n}-3 \beta_{m}+\delta T_{2}$. Then, the system of equations reduces to

$$
\omega_{m} D_{2} a_{m}=F_{m} \sin \left(\gamma_{1}\right)+R_{3} a_{m}^{2} a_{n} \sin \left(\gamma_{2}\right)-\mu_{1} \omega_{m} a_{m}
$$




$$
\begin{aligned}
& \omega_{m} a_{m} D_{2} \gamma_{1}=\omega_{m} a_{m} \alpha+F_{m} \cos \left(\gamma_{1}\right)+R_{3} a_{m}{ }^{2} a_{n} \cos \left(\gamma_{2}\right)+R_{2} a_{m} a_{n}{ }^{2}+R_{1} a_{m}{ }^{3} \\
& \omega_{m} D_{2} a_{n}=-K_{3} a_{m}^{3} \sin \left(\gamma_{2}\right)-\mu_{2} \omega_{n} a_{n} \\
& \omega_{m} a_{n}\left(D_{2} \gamma_{2}-3 D_{2} \gamma_{1}\right)=\omega_{m} a_{n}(\delta-3 \alpha)-K_{3} a_{m}{ }^{3} \cos \left(\gamma_{2}\right)-K_{1} a_{m}{ }^{2} a_{n}-K_{1} a_{n}{ }^{3}
\end{aligned}
$$

The fixed points of the system are obtained by solving the right-hand side of Eq. (25.1)-Eq. (25.4) using an arc-length continuation method [30]. Then, to express the full system solution, the value of the bookkeeping parameter $\varepsilon$ is set equal to unity and we substitute Eq. (14) and Eq. (17) with Eq. (23.1) and Eq. (23.2) yields

$$
\begin{aligned}
y & =y_{s}(x)+a_{m} \phi_{m}(x) \cos \left(\left(\omega_{m}+\alpha\right) t-\gamma_{1}\right)+a_{n} \phi_{n}(x) \cos \left(\left(\omega_{n}+3 \alpha-\delta\right) t+\left(\gamma_{2}-3 \gamma_{1}\right)\right) \\
& +\Pi_{1 m}(x) \frac{a_{m}{ }^{2}}{2} \cos \left(2 \omega_{m} t+2\left(\alpha t-\gamma_{1}\right)\right)+\Pi_{2 m}(x) \frac{a_{m}{ }^{2}}{2} \\
& +\Pi_{1 n}(x) \frac{a_{n}^{2}}{2} \cos \left(2\left(\omega_{n}+3 \alpha-\delta\right) t+2\left(\gamma_{2}-3 \gamma_{1}\right)\right)+\Pi_{2 n}(x) \frac{a_{n}{ }^{2}}{2} \\
& +\Pi_{1 m n}(x) \frac{a_{n} a_{m}}{2} \cos \left(\left(\omega_{n}-\omega_{m}\right) t+(3 \alpha-\delta) t+\left(\gamma_{2}-3 \gamma_{1}\right)-\left(\alpha t-\gamma_{1}\right)\right) \\
& +\Pi_{2 m n}(x) \frac{a_{n} a_{m}}{2} \cos \left(\left(\omega_{n}+\omega_{m}\right) t+(3 \alpha-\delta) t+\left(\gamma_{2}-3 \gamma_{1}\right)+\left(\alpha t-\gamma_{1}\right)\right)
\end{aligned}
$$

The stability of the solution is determined by expanding Eq. (21.1) and Eq. (21.2) into complex Cartesian form by assuming the amplitude to be

$$
\begin{aligned}
& A_{m}=\frac{1}{2}\left(p_{m}-\mathrm{i} q_{m}\right) e^{\mathrm{i} \lambda_{1} T_{2}} \\
& A_{n}=\frac{1}{2}\left(p_{n}-\mathrm{i} q_{n}\right) e^{\mathrm{i} \lambda_{2} T_{2}}
\end{aligned}
$$

The modulation equations are given by 


$$
\begin{aligned}
\omega_{m} D_{2} p_{m}= & -\alpha \omega_{m} q_{m}-R_{2} q_{m} p_{n}{ }^{2}+2 R_{3} p_{m} q_{m} p_{n}-R_{3} p_{m}{ }^{2} q_{n}+R_{3} q_{m}{ }^{2} q_{n}-R_{2} q_{m} q_{n}{ }^{2}-R_{1} p_{m}{ }^{2} q_{m} \\
& -\mu_{1} \omega_{m} p_{m}-R_{1} q_{m}{ }^{3} \\
\omega_{m} D_{2} q_{m}= & \alpha \omega_{m} p_{m}+F_{m}+R_{2} p_{m} q_{n}{ }^{2}+2 R_{3} p_{m} q_{m} q_{n}-R_{3} q_{m}{ }^{2} p_{n}+R_{3} p_{m}{ }^{2} p_{n}+R_{2} p_{m} p_{n}{ }^{2}+R_{1} p_{m} q_{m}{ }^{2} \\
& +R_{1} p_{m}{ }^{3}-\mu_{1} \omega_{m} q_{m} \\
\omega_{m} D_{2} p_{n}= & -3 \alpha \omega_{m} q_{n}-\delta \omega_{m} q_{n}+K_{1} p_{m}{ }^{2} q_{n}-K_{1} q_{m}{ }^{2} q_{n}-3 K_{3} p_{m}{ }^{2} q_{m}+K_{3} q_{m}{ }^{3}-K_{1} p_{n}{ }^{2} q_{n}-K_{1} q_{n}{ }^{3} \\
& -\mu_{2} \omega_{n} p_{n} \\
\omega_{m} D_{2} q_{n}= & 3 \alpha \omega_{m} p_{n}-\delta \omega_{m} p_{n}+K_{1} q_{m}{ }^{2} p_{n}+K_{1} p_{m}{ }^{2} p_{n}-3 K_{3} p_{m} q_{m}{ }^{2}+K_{3} p_{m}{ }^{3}+K_{1} p_{n} q_{n}{ }^{2}+K_{1} p_{n}{ }^{3} \\
& -\mu_{2} \omega_{n} q_{n}
\end{aligned}
$$

where $\lambda_{1}=\alpha$ and $\lambda_{2}=3 \alpha-\delta$. The stability of the fixed points is determined by examining the eigenvalue of the Jacobain of the system of Eq. (28.1)-Eq. (28.4).

\section{Results and Discussion}

For the numerical results, the data in Table 1 are utilized.

Table 1. Values of the parameters used in the calculations.

\begin{tabular}{|c|c|c|c|c|c|c|}
\hline Parameter & $\begin{array}{c}\text { Outside } \\
\text { Diameter } \\
\boldsymbol{D}\end{array}$ & $\begin{array}{c}\text { Inside } \\
\text { Diameter } \\
\boldsymbol{D}_{\boldsymbol{i}}\end{array}$ & $\begin{array}{c}\text { Modulus of } \\
\text { Elasticity } \\
\boldsymbol{E}\end{array}$ & $\begin{array}{c}\text { Density of } \\
\text { Riser } \\
\boldsymbol{\rho}_{\boldsymbol{p}}\end{array}$ & $\begin{array}{c}\text { Density of Sea } \\
\text { Water } \\
\boldsymbol{\rho}_{\boldsymbol{e}}\end{array}$ & $\begin{array}{c}\text { Depth of } \\
\text { Sea }\end{array}$ \\
\hline Value & $0.26 \mathrm{~m}$ & $0.2 \mathrm{~m}$ & $207 \mathrm{GPa}$ & $7850 \mathrm{Kg} / \mathrm{m}^{3}$ & $1025 \mathrm{Kg} / \mathrm{m}^{3}$ & $150 \mathrm{~m}$ \\
\hline
\end{tabular}

The geometric properties of the riser are similar to those analyzed in [31]; however, the influence of the static deflection is accounted for in this analysis, which enriches the dynamic behavior of the system. In all the MTS results that follows, the value of $\varepsilon$ is set to unity.

\subsection{Frequency Results}


The natural frequencies and mode shapes of the riser are studied by solving the linear eigenvalue problem in Eq. (8), using fifteen unforced beam mode shapes, via the Galerkin method. Depicted in Fig. 2 is the variation of the lowest five natural frequencies against the configuration angle.
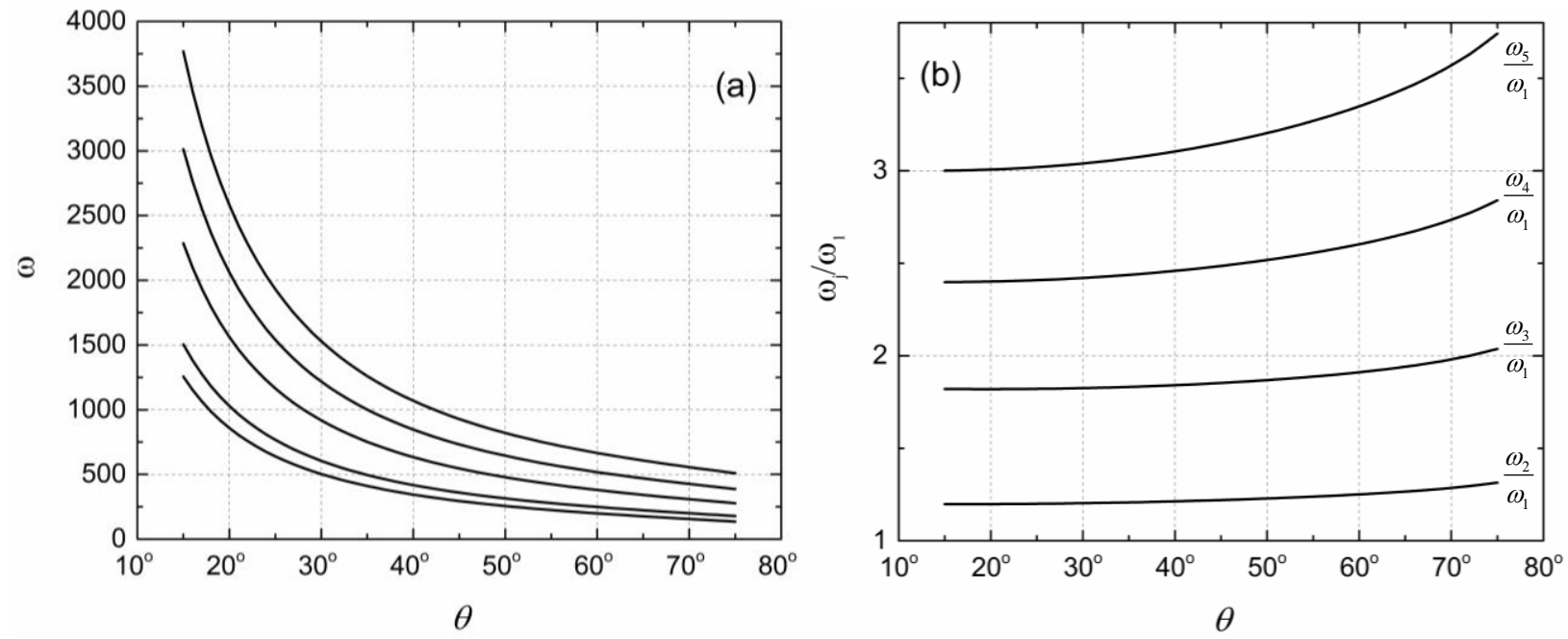

Figure 2. Natural frequency values at different inclination angles at $\bar{T}=1.2 \bar{\sigma}$. (a) Lowest five natural frequencies. (b) Normalized natural frequency values with respect to the first natural frequency.

At a fixed value of applied tension ratio, $T_{r}=\bar{T} / \bar{\sigma}=1.2$, increasing the configuration angle decreases the values of the natural frequency due to the decrease in submerged length and weight. Also, the influence of the distributed weight load becomes less at higher configuration angles. Because the riser structure is dominated by the applied tension, the observed mode shapes are near symmetric. Therefore, we seek tension values such that the activation of internal resonance between the higher order symmetric mode and the first mode requires less amount of forcing. This result is noticed when plotting frequency ratios with respect to the first natural frequency in Fig. 2b. In order to realize a three-to-one ratio, one needs to tune the configuration angle. We note the three-to-one ratio between the fifth and the first modes. Therefore, the 
configuration angle leading to this ratio is fixed and we tune the tension in and out of the internal resonance to study the interaction. The values extracted from Fig. $2 b$ are shown in Table 2.

Table 2. Values of extracted frequencies leading to a 3:1 ratio between the fifth and the first modes.

\begin{tabular}{ccc}
\hline $\boldsymbol{\theta}$ & $\omega_{1}$ & $\omega_{5}$ \\
\hline $15^{\circ}$ & 1256.68 & 3769.46 \\
\hline
\end{tabular}

\subsection{Perturbation Results for the Three-To-One Internal Resonance}

Next, we study the response of the riser when the three-to-one internal resonance is activated. Since the activation of the internal resonance occurs at the third order, the nature of the shape functions in Eq. (18) becomes of great importance. The shape functions are plotted in Fig. 3.
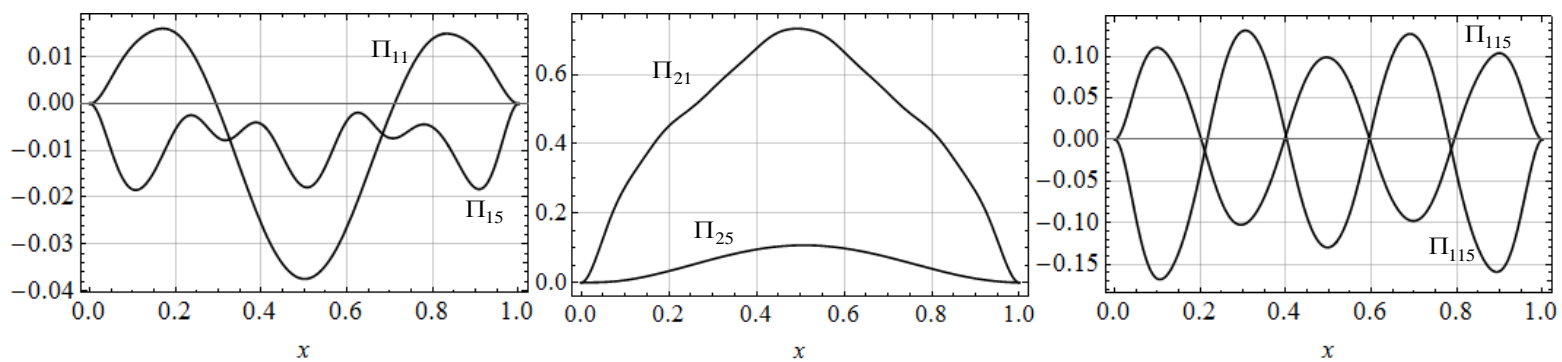

Figure 3. Plot of the particular solutions $\Pi_{11}, \Pi_{15}, \Pi_{21}, \Pi_{25}, \Pi_{115}$ and $\Pi_{215}$ at $\bar{T}=1.2 \bar{\sigma}$ and $\theta=15^{\circ}$.

The interaction of the shape functions shown in Fig. 3 with the static solution and the mode shapes participating in the internal resonance determines the condition to activate the internal resonance. In addition, the strength of this activation is affected by how strong or weak are the coupling coefficients $R_{2}, R_{3}, K_{2}$, and $K_{3}$ in Eq. (21.1) and Eq. (21.2). The values of these coefficients corresponding to tension ratio of 1.2 are shown in Table 3.

Table 3. The values of coefficients $R_{i}, K_{i}$ in Eq. (23). 


\begin{tabular}{cccccc}
\hline$R_{1}$ & $R_{2}$ & $R_{3}$ & $K_{1}$ & $K_{2}$ & $K_{3}$ \\
\hline 445.63 & 5405.89 & 239.61 & -69755.11 & 5415.11 & 80.77 \\
\hline
\end{tabular}

Furthermore, we validate the solution obtained by MTS by solving the nonlinear Eq. (6) using five mode shapes extracted from Eq. (8) in the Galerkin procedure. The multiple scales solution is obtained by solving the amplitude modulations Eq. (25) and, then, substituting the obtained fixed points in Eq. (26) to get the full solution. The result of the comparison is depicted in Fig. 4.

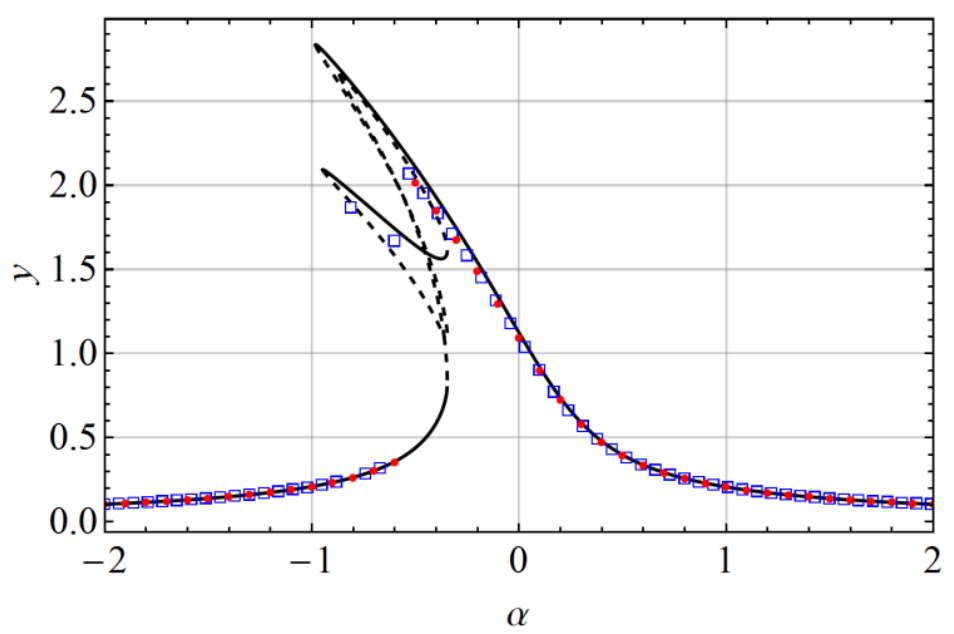

Figure 4. Frequency response curves showing the maximum deflection $y$ as a function of the detuning parameter $\alpha$. $\theta=15^{\circ}, \mu_{1}=0.08, \mu_{2}=0.08, F_{m}=169.58$. (—) Stable fixed points, $(--)$ Unstable fixed points perturbation method. ( $\square$ ) Solution by long time integration (forward sweep). ( ० ) Solution by long time integration (backward sweep).

As observed from Fig. 4 the Galerkin solution in the forward and backward sweeps agrees well with the solution obtained by the multiple scales method. The solution of the multiple scales shows the emergence of a new solution between the softening upper branch and the lower branch yielding a tristate between $\alpha=-0.34$ and $\alpha=-1$. The interaction of the middle stable solution with the unstable solutions implies that the basin of attraction might be weak. This is confirmed by the fact that catching this solution using the Galerkin method was very hard and was done by 
adjusting manually initial conditions until the solution converged to the middle branch. A similar behavior is observed in the upper branch where the solution does not have sufficient basin of attraction to remain in that branch and jumps to the lower solution branch.

It is noteworthy that the current velocity required to produce the amount of force used in this simulation corresponds to $U=0.0371 \mathrm{~m} / \mathrm{s}$. This velocity value, compared to practical values inducing the vortex induced vibrations (VIV), suggests that such type of loading with internal resonance is likely to occur in real life applications. A further examination is carried out by plotting the response of the first and fifth mode amplitudes in Fig. 5.
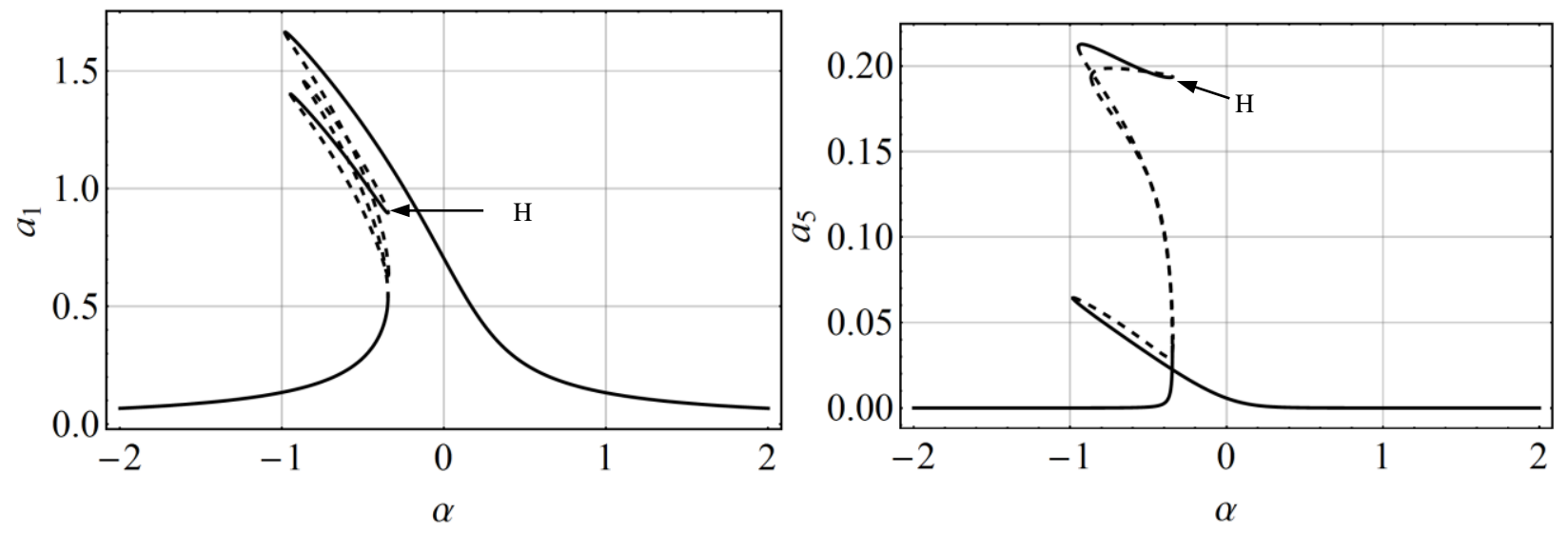

Figure 5. Frequency response curves showing the first and fifth modes as a function of the detuning parameter $\alpha$. $\theta=15^{\circ}, \mu_{1}=0.08, \mu_{2}=0.08, F_{m}=169.58$. (—) Stable fixed solutions, $(--)$ unstable fixed solution. H denotes the point of a Hopf bifurcation.

Although, the activation of internal resonance yields a weak contribution from the fifth modes, it alters the response of the first mode and affects the dynamic behavior of the response when the interaction region is near the hysteresis zone. The first mode amplitude experiences a saddle node jump from the lower branch to the middle branch where it passes through a reverse Hopf bifurcation. Then, the system experiences another saddle node jump from the middle branch to the upper branch (softening branch) where the solution diminishes upon further sweeping the frequency. The interaction of the saddle node jump with the middle solution branch suggests that 
the solution have two possibilities, either a direct jump from the lower branch to the upper branch or a jump from the lower branch to the middle branch. A similar behavior is observed in the response of the fifth mode where three solutions exist. However, the interaction from the saddle node jump with the solution in this case is less present. The time histories of these results based on (MTS) solution are plotted in Fig. 6.

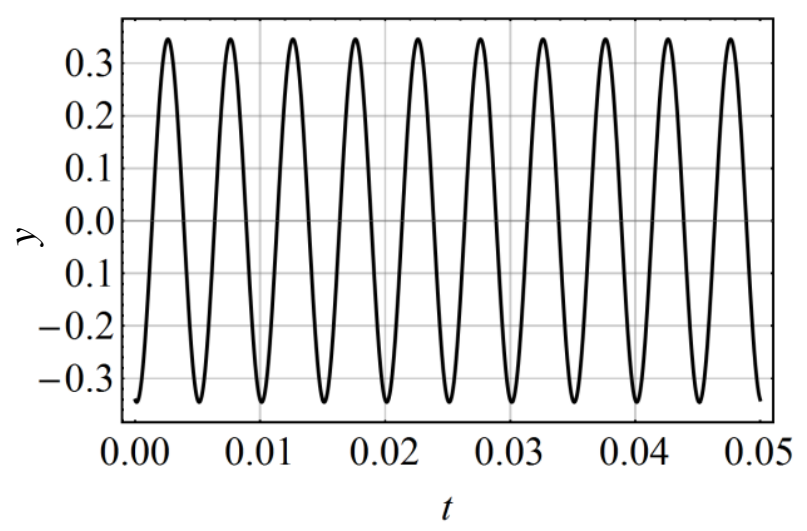

(a)

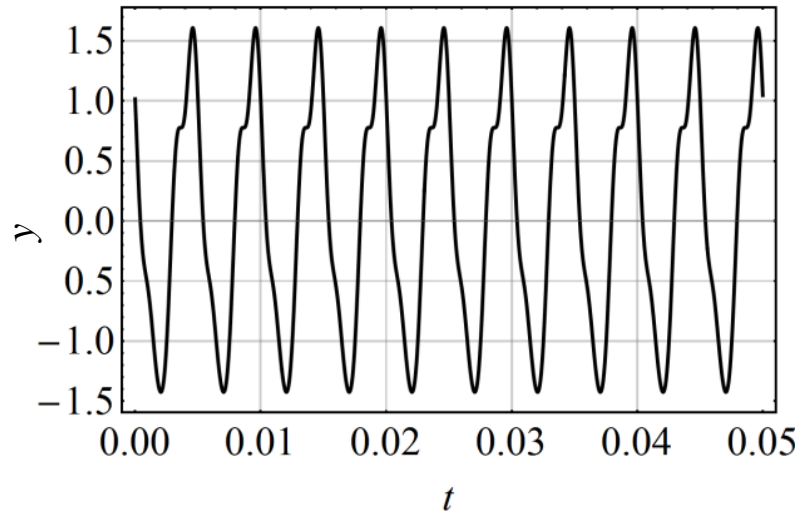

(b)

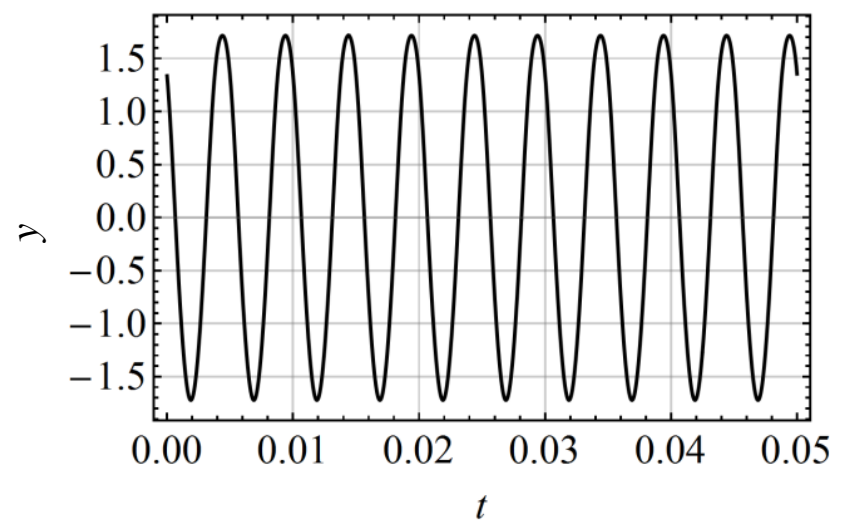

(c)

Figure 6. Time history for $\theta=15^{\circ}, \mu_{1}=0.08, \mu_{2}=0.08, F_{m}=169.58$. (a) Lower branch, $\alpha=-0.6$ from Fig. 4 . (b) Middle branch, $\alpha=-0.35$ from Fig. 4. (c) Upper branch, $\alpha=-0.4$ from Fig. 4

The time histories presented in Fig. 6 indicate that the solutions in the lower branch and upper branch are dominated by a single period response while the time history of the solution in the middle branch is a two-period response. This indicates that the internal resonance is of stronger influence in the middle branch. The time history of temporal coefficients of each mode is plotted 
using the Galerkin solution for further comparison and confirmation in Fig. 7 and Fig. 8. The time history in Fig. 7 and the corresponding power spectral densities confirm the results obtain in multiple scales method and show that the contribution of the fifth mode is very weak away from the internal resonance regime, middle branch. The amplitude increases when the response is at the middle branch. We note that the influence of the first mode on the fifth mode is stronger than the influence of the fifth on the first mode because the excitation is around the first primary resonance.

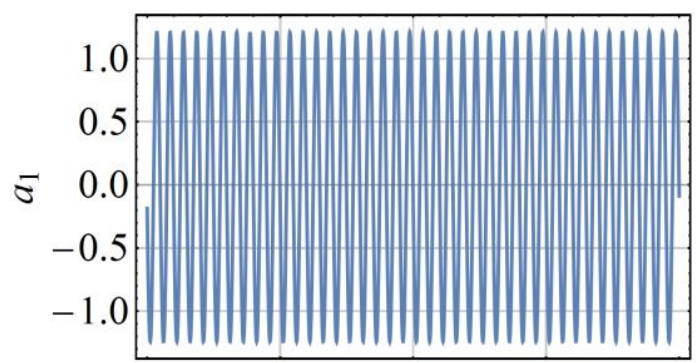

$199.80 \quad 199.85 \quad 199.90 \quad 199.95 \quad 200.00$

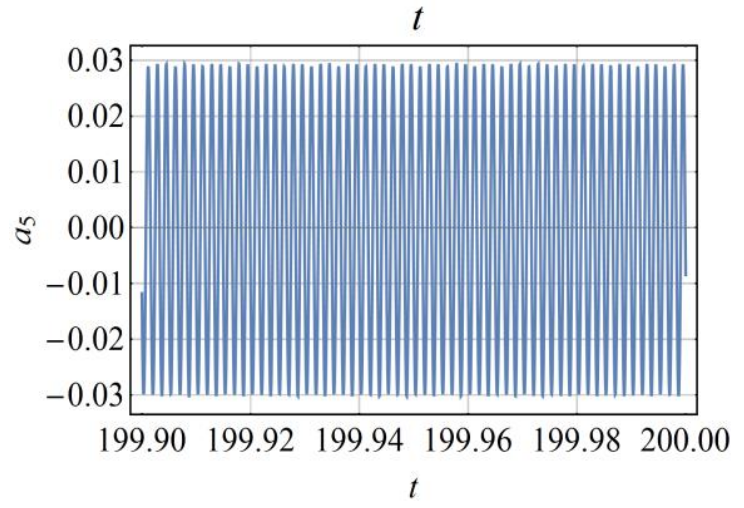

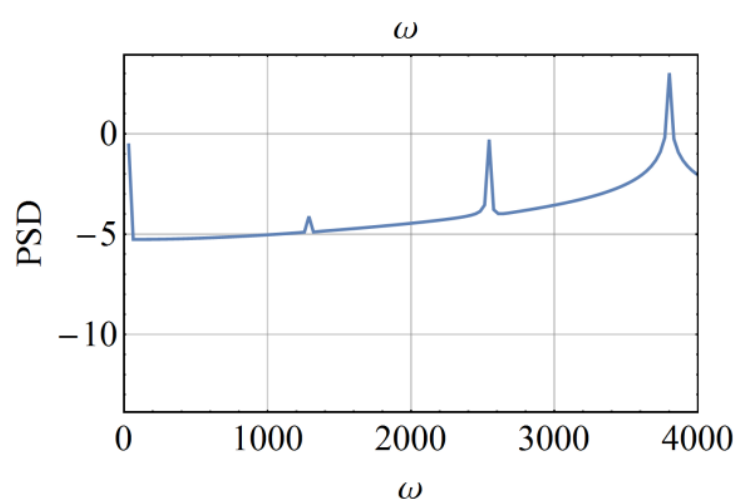

Figure 7. Dynamical solution from the Galerkin model and the corresponding Fourier spectrum. $\theta=15^{\circ}, \bar{T}=1.2 \bar{\sigma}$, $\mu_{1}=0.08, \quad \mu_{2}=0.08, F_{m}=169.58, \alpha=-0.45$, from the lower branch of the responses in Fig. 5 . 

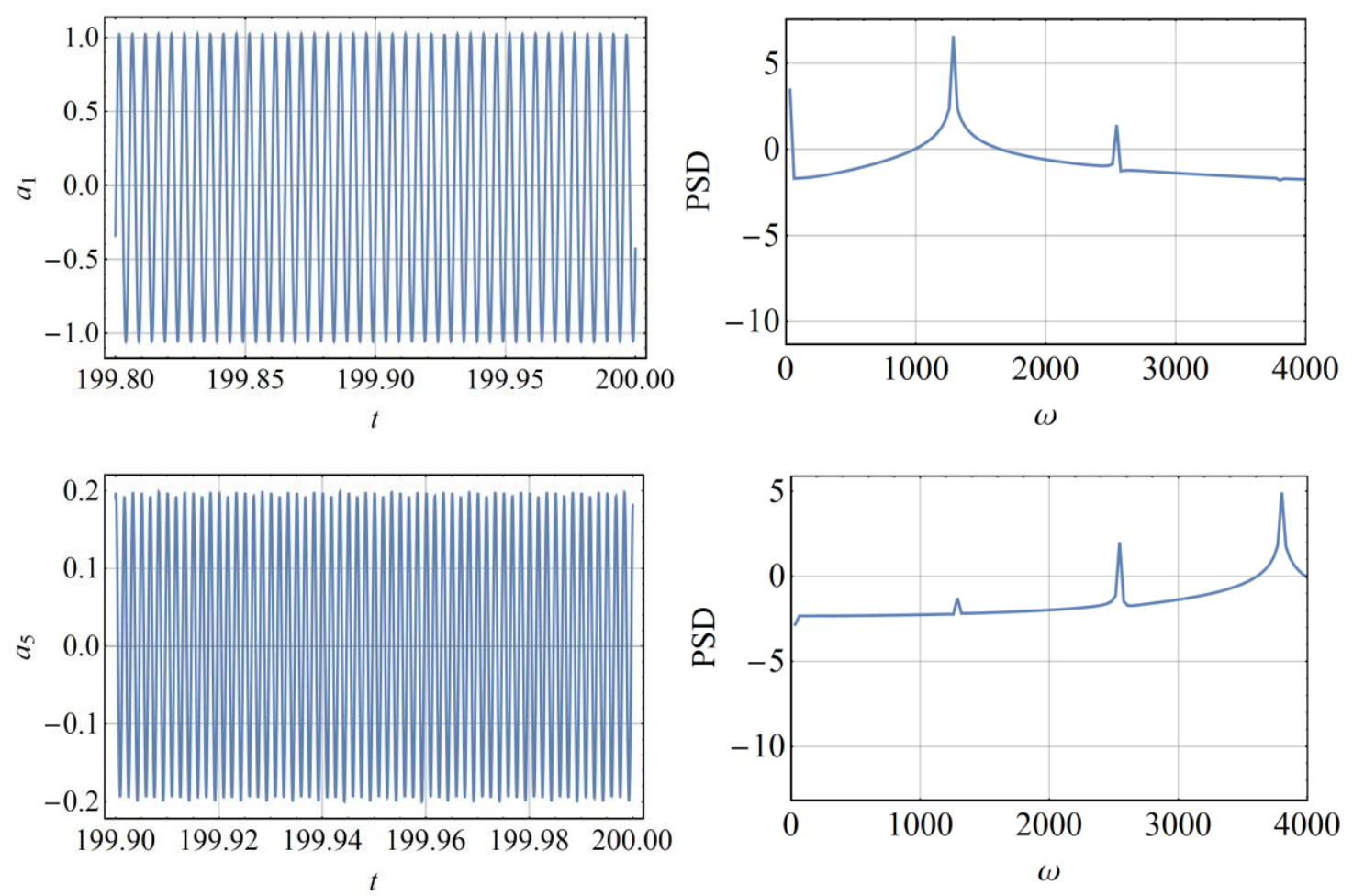

Figure 8. Dynamical solution from the Galerkin and the corresponding Fourier spectrum. $\theta=15^{\circ}, \bar{T}=1.2 \bar{\sigma}$, $\mu_{1}=0.08, \mu_{2}=0.08, F_{m}=169.58, \alpha=-0.5$, from the middle branch of the responses in Fig. 5 .

Furthermore, the power spectral densities in Fig. 7 and Fig. 8 show the $2 \omega_{1}$ component. Depending on the level of interaction between the first and fifth modes in the three-to-one internal resonance at different values of excitation frequency, the secondary frequency terms in Eq. (26) pertaining to the particular solution at the second order can be magnified depending on the level of interaction. In the presented case in Fig. 7 and Fig. 8, the intensity of the $2 \omega_{1}$ frequency in the fifth mode increases from below zero at the lower branch of the solution in Fig. 7 to a value above zero in the middle branch in Fig. 8. This indicates that the contribution of the particular solution to activate the three-to-one internal resonance is considerable. The intensity of such contribution is based on the force amplitude applied to the system. To investigate the influence of the force amplitude, the force response curves are plotted in Fig. 9. 

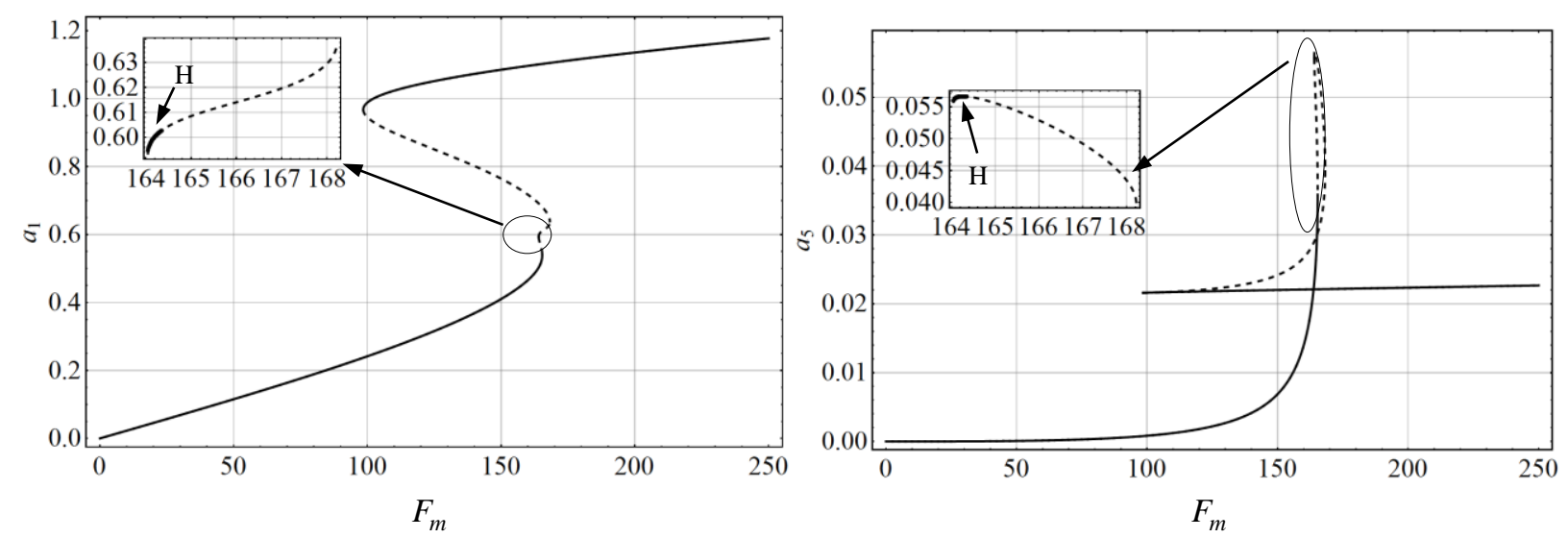

Figure 9. Force response curves showing the first and fifth mode amplitudes as a function of forcing. $\theta=15^{\circ}$, $\mu_{1}=0.08, \mu_{2}=0.08, \alpha=-0.34$. (-) Stable fixed solutions, $\left(-\boldsymbol{C}^{-}\right.$) unstable fixed solution and $\mathrm{H}$ denotes the point of a Hopf bifurcation. Insets: magnified response curves around the bifurcation point.

The response curves shown in Fig. 9 are plotted at $\alpha=-0.34$, which is near the Hopf bifurcation to investigate how the response behavior changes with increasing forcing amplitude. From Fig. 9a we notice that the first mode amplitude increases upon increasing the force amplitude. Then, the amplitude passes through a Hopf bifurcation leading to the saddle node bifurcation. The response after the saddle node bifurcation is shown to be stable. Likewise, the fifth mode amplitude response exhibits a Hopf and saddle node bifurcations with increasing the forcing amplitude. After that, the response of the fifth mode amplitude shows saturation where the response remains nearly unchanged with increasing force amplitude.

Next, we change the applied tension ratio slightly to $\bar{T}=1.21 \bar{\sigma}$, and hence change the natural frequencies ratio to $\omega_{5} / \omega_{1} \approx 2.99$, and we plot the frequency response curves of the first and fifth modes. The results are depicted in Fig. 10. 

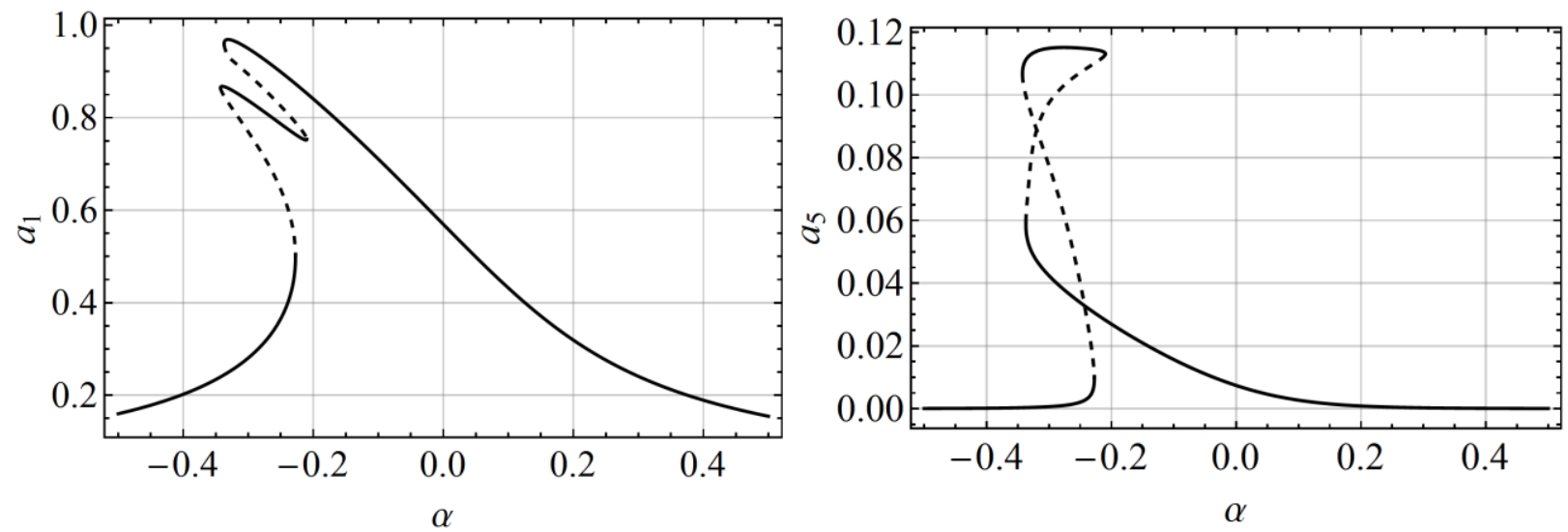

Figure 10. Frequency response curves showing the first and fifth modes as a function of the detuning parameter $\alpha$. $\theta=15^{\circ}, \bar{T}=1.21 \bar{\sigma}, \mu_{1}=0.08, \mu_{2}=0.08, F_{m}=100$. (—) Stable solutions, $(--)$ unstable solutions.

The response of the first and fifth modes show that the interaction presented in Fig. 10 is less than the previously shown results in Fig. 5. The amplitude of the first mode response shows saddle node jumps between the lower, middle, and upper solution branches but they do not exhibit any Hopf bifurcation or intersecting/crossing behavior. Likewise, the response of the fifth mode is shifted towards the lower frequency values and shows less amplitude upon activation. This is because the activation of this response requires less amount of forcing compared to the previous results. To investigate the influence of forcing amplitude, we plot the force response curves corresponding to this case in Fig. 11.
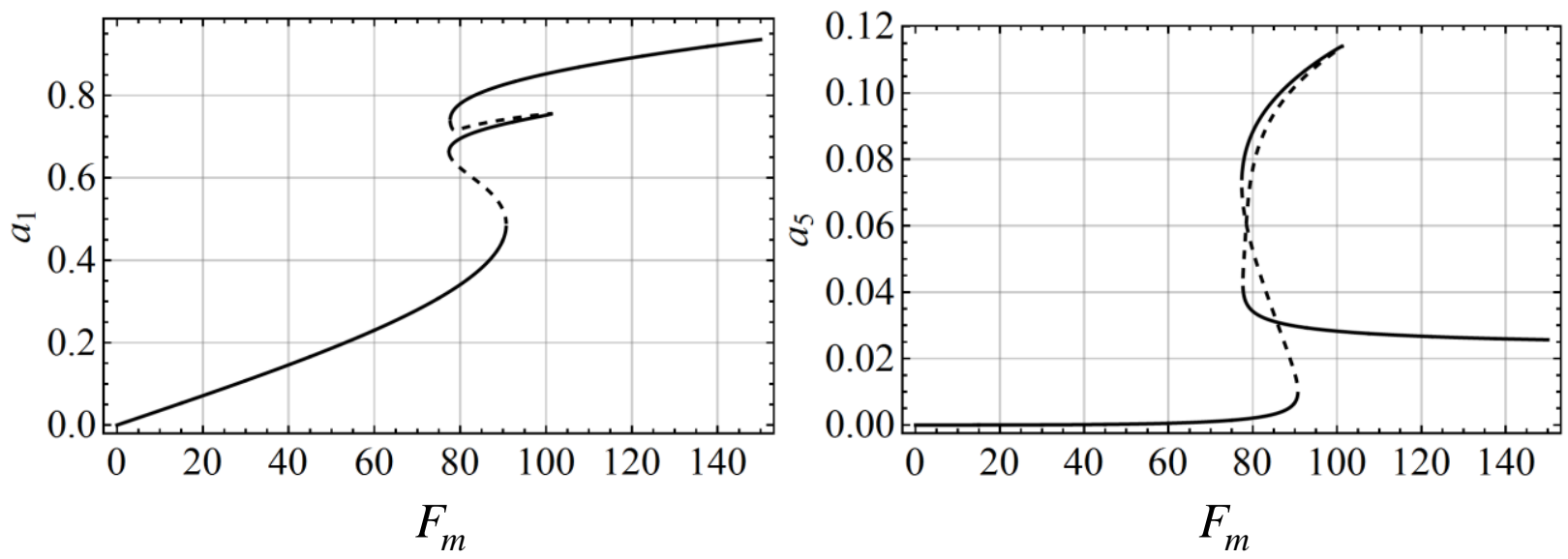
Figure 11. Force response curve showing the first and fifth modes amplitudes as a function of forcing. $\theta=15^{\circ}$,

$\bar{T}=1.21 \bar{\sigma}, \mu_{1}=0.08, \mu_{2}=0.08, \alpha=-0.30$. (-) Stable solutions, $(--)$ unstable solutions, and $\mathrm{H}$ denotes the point of a Hopf bifurcation.

The force amplitude curves in Fig. 11 show clearly three stables states in the forcing range from 70 to 100 . The first mode amplitude response increases with increasing forcing until the activation region where the solution experiences a saddle node jump to an intermediate branch and another saddle node jump to the upper branch. Then, the response amplitude increases with increasing forcing. The response of the fifth mode shows a similar response with the existence of three stable states. In addition, the saturation of the amplitude occurs at an amplitude equivalent to that observed in the previous case.

\section{Conclusions}

In this paper, we analyzed the three-to-one internal resonance of an inclined riser under harmonic excitations between the first and fifth modes. The riser model accounts for mid-plane stretching, self-weight axial load, and the initial static deflection. After that, the multiple time scales method was utilized to study the nonlinear response of the riser considering the internal resonance interaction. The results showed that the response of the riser featured nonlinear saddle node and Hopf bifurcations. In addition, the response due to the internal resonance activation introduced an intermediate branch despite the fact that the influence of the internal resonance on the fifth mode response is very weak. The existence of such response characteristic makes the riser structure susceptible to fatigue damage due to the presence of a higher frequency component in the response of the structure and the presence of jumps between the solutions. A better understanding of such interaction is needed in future considering the coupled fluid structure interaction accounting for vortex induced vibration forces. 


\section{Acknowledgements}

We acknowledge the financial support of King Abdullah Univeristy of Science and Technology and Saudi Aramco.

\section{Conflict of interest}

The authors declare that they have no conflict of interest.

Appendix A. Definition of the coefficients $\psi_{1}(x), \psi_{2}(x), \psi_{3}(x), \psi_{4}(x), \psi_{5}(x)$ and $\psi_{6}(x)$ in Eq.

$$
\begin{aligned}
\psi_{1}(x) & =2 \eta\left(\int_{0}^{1} \Pi_{1 m}^{\prime} \phi_{m}^{\prime} \mathrm{d} x\right) y_{s}^{\prime \prime}+2 \eta\left(\int_{0}^{1} \Pi_{2 m}^{\prime} \phi_{m}^{\prime} \mathrm{d} x\right) y_{s}^{\prime \prime}+2 \eta\left(\int_{0}^{1} y_{s}^{\prime} \phi_{m}^{\prime} \mathrm{d} x\right) \Pi_{1 m}{ }^{\prime \prime}+2 \eta\left(\int_{0}^{1} y_{s}^{\prime} \phi_{m}^{\prime} \mathrm{d} x\right) \Pi_{2 m}{ }^{\prime \prime} \\
& +2 \eta\left(\int_{0}^{1} y_{s}^{\prime} \Pi_{1 m}^{\prime} \mathrm{d} x\right) \phi_{m}^{\prime \prime}+2 \eta\left(\int_{0}^{1} y_{s}^{\prime} \Pi_{2 m}^{\prime} \mathrm{d} x\right) \phi_{m}^{\prime \prime}+3 \eta\left(\int_{0}^{1}\left(\phi_{m}^{\prime}\right)^{2} \mathrm{~d} x\right) \phi_{m}^{\prime \prime}
\end{aligned}
$$

$$
\begin{aligned}
\psi_{2}(x) & =2 \eta\left(\int_{0}^{1} \Pi_{2 n}^{\prime} \phi_{m}^{\prime} \mathrm{d} x\right) y_{s}^{\prime \prime}+2 \eta\left(\int_{0}^{1} \Pi_{1 m n} \phi_{n}^{\prime} \mathrm{d} x\right) y_{s}^{\prime \prime}+2 \eta\left(\int_{0}^{1} \Pi_{2 m n}^{\prime} \phi_{n}^{\prime} \mathrm{d} x\right) y_{s}^{\prime \prime} \\
& +2 \eta\left(\int_{0}^{1} y_{s}^{\prime} \phi_{m}^{\prime} \mathrm{d} x\right) \Pi_{2 n}^{\prime \prime}+2 \eta\left(\int_{0}^{1} y_{s}^{\prime} \phi_{n}^{\prime} \mathrm{d} x\right) \Pi_{1 m n}{ }^{\prime \prime}+2 \eta\left(\int_{0}^{1} y_{s}^{\prime} \phi_{n}^{\prime} x\right) \Pi_{2 m n}{ }^{\prime \prime}+2 \eta\left(\int_{0}^{1} y_{s}^{\prime} \Pi_{2 n}^{\prime} \mathrm{d} x\right) \phi_{m}^{\prime \prime} \\
& +2 \eta\left(\int_{0}^{1}\left(\phi_{n}^{\prime}\right)^{2} \mathrm{~d} x\right) \phi_{m}^{\prime \prime}+2 \eta\left(\int_{0}^{1} y_{s}^{\prime} \Pi_{1 m n}{ }^{\prime} \mathrm{d} x\right) \phi_{n}^{\prime \prime}+2 \eta\left(\int_{0}^{1} y_{s}^{\prime} \Pi_{2 m n}{ }^{\prime} \mathrm{d} x\right) \phi_{n}^{\prime \prime}+4 \eta\left(\int_{0}^{1} \phi_{m}^{\prime} \phi_{n}^{\prime} \mathrm{d} x\right) \phi_{n}^{\prime \prime}
\end{aligned}
$$

$$
\begin{aligned}
\psi_{3}(x) & =2 \eta\left(\int_{0}^{1} \Pi_{1 n}^{\prime} \phi_{n}^{\prime} \mathrm{d} x\right) y_{s}^{\prime \prime}+2 \eta\left(\int_{0}^{1} \Pi_{2 n}^{\prime} \phi_{n}^{\prime} \mathrm{d} x\right) y_{s}^{\prime \prime}+2 \eta\left(\int_{0}^{1} y_{s}^{\prime} \phi_{n}^{\prime} \mathrm{d} x\right) \Pi_{1 n}^{\prime \prime}+2 \eta\left(\int_{0}^{1} y_{s}^{\prime} \phi_{n}^{\prime} \mathrm{d} x\right) \Pi_{2 n}{ }^{\prime \prime} \\
& +2 \eta\left(\int_{0}^{1} y_{s}^{\prime} \Pi_{1 n}^{\prime} \mathrm{d} x\right) \phi_{n}^{\prime \prime}+2 \eta\left(\int_{0}^{1} y_{s}^{\prime} \Pi_{2 n}^{\prime} \mathrm{d} x\right) \phi_{n}^{\prime \prime}+3 \eta\left(\int_{0}^{1}\left(\phi_{n}^{\prime}\right)^{2} \mathrm{~d} x\right) \phi_{n}^{\prime \prime}
\end{aligned}
$$




$$
\begin{aligned}
\psi_{4}(x)= & 2 \eta\left(\int_{0}^{1} \Pi_{1 m n}{ }^{\prime} \phi_{m}^{\prime} \mathrm{d} x\right) y_{s}^{\prime \prime}+2 \eta\left(\int_{0}^{1} \Pi_{2 m n}^{\prime} \phi_{m}^{\prime} \mathrm{d} x\right) y_{s}^{\prime \prime}+2 \eta\left(\int_{0}^{1} \Pi_{2 m}^{\prime} \phi_{n}^{\prime} \mathrm{d} x\right) y_{s}^{\prime \prime}+4 \eta\left(\int_{0}^{1} \phi_{m}^{\prime} \phi_{n}^{\prime} \mathrm{d} x\right) \phi_{m}^{\prime \prime} \\
& +2 \eta\left(\int_{0}^{1} y_{s}^{\prime} \Pi_{1 m n}^{\prime} \mathrm{d} x\right) \phi_{m}^{\prime \prime}+2 \eta\left(\int_{0}^{1} y_{s}^{\prime} \Pi_{2 m n}^{\prime} \mathrm{d} x\right) \phi_{m}^{\prime \prime}+2 \eta\left(\int_{0}^{1} y_{s}^{\prime} \Pi_{2 m}^{\prime} \mathrm{d} x\right) \phi_{n}^{\prime \prime}+2 \eta\left(\int_{0}^{1}\left(\phi_{m}^{\prime}\right)^{2} \mathrm{~d} x\right) \phi_{n}^{\prime \prime} \\
& +2 \eta\left(\int_{0}^{1} y_{s}^{\prime} \phi_{n}^{\prime} \mathrm{d} x\right) \Pi_{2 m}^{\prime \prime}+2 \eta\left(\int_{0}^{1} y_{s}^{\prime} \phi_{m}^{\prime} \mathrm{d} x\right) \Pi_{1 m n}^{\prime \prime}+2 \eta\left(\int_{0}^{1} y_{s}^{\prime} \phi_{m}^{\prime} \mathrm{d} x\right) \Pi_{2 m n}^{\prime \prime} \\
\psi_{5}(x)= & 2 \eta y_{s}^{\prime \prime}\left(\int_{0}^{1} \phi_{m}^{\prime} \Pi_{1 m n}^{\prime} \mathrm{d} x\right)+2 \eta y_{s}^{\prime \prime}\left(\int_{0}^{1} \Pi_{1 m}^{\prime} \phi_{n}^{\prime} \mathrm{d} x\right)+2 \eta \Pi_{1 m}^{\prime \prime}\left(\int_{0}^{1} \phi_{n}^{\prime} y_{s}^{\prime} \mathrm{d} x\right)+2 \eta \Pi_{1 m n}^{\prime \prime}\left(\int_{0}^{1} \phi_{m}^{\prime} y_{s}^{\prime} \mathrm{d} x\right) \\
& +2 \eta \phi_{m}^{\prime \prime}\left(\int_{0}^{1} y_{s}^{\prime} \Pi_{1 m n}^{\prime} \mathrm{d} x\right)+2 \eta \phi_{n}^{\prime \prime}\left(\int_{0}^{1} \Pi_{1 m}^{\prime} y_{s}^{\prime} \mathrm{d} x\right)+2 \eta \phi_{m}^{\prime \prime}\left(\int_{0}^{1} \phi_{m}^{\prime} \phi_{n}^{\prime} \mathrm{d} x\right)+\eta \phi_{n}^{\prime \prime}\left(\int_{0}^{1}\left(\phi_{m}^{\prime}\right)^{2} \mathrm{~d} x\right) \\
\psi_{6}(x)= & 2 \eta y_{s}^{\prime \prime}\left(\int_{0}^{1} \Pi_{1 m}^{\prime} \phi_{m}^{\prime} \mathrm{d} x\right)+2 \eta \Pi_{1 m}^{\prime \prime}\left(\int_{0}^{1} \phi_{m}^{\prime} y_{s}^{\prime} \mathrm{d} x\right)+2 \eta \phi_{m}^{\prime \prime}\left(\int_{0}^{1} \Pi_{1 m}^{\prime} y_{s}^{\prime} \mathrm{d} x\right)+\eta \phi_{m}^{\prime \prime}\left(\int_{0}^{1}\left(\phi_{m}^{\prime}\right)^{2} \mathrm{~d} x\right)
\end{aligned}
$$

Appendix B. Proof of self-adjoint property of the linear eigenvalue problem

In this section, we provide the proof of the self-adjointness of the eigenvalue problem in Eq. (8). To ensure that $\phi_{j}(x) e^{ \pm i \omega_{j} T_{0}}$ satisfies the adjoint problem, we substitute it in the linear homogenous Eq. (8) and the result is integrated by parts from $x=0$ to $x=1$ reducing to 


$$
\begin{aligned}
& {\left[\phi_{j} \phi_{m}^{\prime \prime \prime}-\phi_{j}^{\prime} \phi_{m}^{\prime \prime}+\phi_{j}^{\prime \prime} \phi_{m}^{\prime}-\phi_{j}^{\prime \prime \prime} \phi_{m}+\overline{T_{s}} \phi_{j} \phi_{m}{ }^{\prime}+\bar{T}_{s} \phi_{j}^{\prime} \phi_{m}-\bar{\sigma} x \phi_{j} \phi_{m}^{\prime}+\bar{\sigma}\left(x \phi_{j}\right)^{\prime} \phi_{m}-\bar{\sigma} \phi_{j} \phi_{m}\right]_{0}^{1}} \\
& +\left[\phi_{j} \phi_{n}^{\prime \prime \prime}-\phi_{j}^{\prime} \phi_{n}^{\prime \prime}+\phi_{j}^{\prime \prime} \phi_{n}^{\prime}-\phi_{j}^{\prime \prime \prime} \phi_{n}+\bar{T}_{s} \phi_{j} \phi_{n}^{\prime}+\bar{T}_{s} \phi_{j}^{\prime} \phi_{n}-\bar{\sigma} x \phi_{j} \phi_{n}^{\prime}+\bar{\sigma}\left(x \phi_{j}\right)^{\prime} \phi_{n}-\bar{\sigma} \phi_{j} \phi_{n}\right]_{0}^{1} \\
& +2 \eta\left[\phi_{j} y_{s}^{\prime}\left(\int_{0}^{1} y_{s}^{\prime \prime} \phi_{m} \mathrm{~d} x\right)+\phi_{m} y_{s}^{\prime}\left(\int_{0}^{\prime}\left(\int_{0}^{1} y_{s}^{\prime} y_{j}^{\prime \prime} \phi_{n} \mathrm{~d} x\right)\right]_{0}^{1}+2 \eta\left[+\phi_{n} y_{s}^{\prime}\left(\int_{0}^{1} y_{s}^{\prime} \phi_{j} \mathrm{~d} x\right)\right]_{0}\right. \\
& +\int_{0}^{1} \phi_{m}\left[-\omega_{m}^{2} \phi_{j}+\phi_{j}^{i v}+\bar{T}_{s} \phi_{j}^{\prime \prime}-\bar{\sigma}\left(x \phi_{j}\right)^{\prime \prime}+\bar{\sigma} \phi_{j}^{\prime}-2 \eta y_{s}^{\prime \prime}\left(\int_{0}^{1} y_{s}^{\prime} \phi_{j} \mathrm{~d} x\right)\right] d x \\
& +\int_{0}^{1} \phi_{n}\left[-\omega_{n}^{2} \phi_{j}+\phi_{j}^{i v}+\bar{T}_{s} \phi_{j}^{\prime \prime}-\bar{\sigma}\left(x \phi_{j}\right)^{\prime \prime}+\bar{\sigma} \phi_{j}^{\prime}-2 \eta y_{s}^{\prime \prime}\left(\int_{0}^{1} y_{s}^{\prime} \phi_{j} \mathrm{~d} x\right)\right] d x=0
\end{aligned}
$$

We note from the expression in Eq. (B.1) that the solution $\phi_{j}(x) e^{ \pm i \omega_{j} T_{0}}$ satisfies Eq. (8) given by the eigenvalue problem and it is self-adjoint.

\section{References}

[1] N.D.P. Barltrop, A.J. Adams, 7 - Vortex-induced vibrations, in: N.D.P. Barltrop, A.J. Adams (Eds.) Dynamics of Fixed Marine Structures (Third Edition), Butterworth-Heinemann, 1991, pp. 345-394.

[2] A. Mehmood, A. Abdelkefi, M.R. Hajj, A.H. Nayfeh, I. Akhtar, A.O. Nuhait, Piezoelectric energy harvesting from vortex-induced vibrations of circular cylinder, Journal of Sound and Vibration, 332 (2013) 4656-4667.

[3] W. Lacarbonara, H.N. Arafat, A.H. Nayfeh, Non-linear interactions in imperfect beams at veering, International Journal of Non-Linear Mechanics, 40 (2005) 987-1003.

[4] A.H. Nayfeh, B. Balachandran, Modal Interactions in Dynamical and Structural Systems, Applied Mechanics Reviews, 42 (1989) S175-S201.

[5] A.H. Nayfeh, Nonlinear Interactions: Analytical, Computational, and Experimental Methods, Wiley, 2000.

[6] G.F. Clauss, H. Weede, A. Saroukh, Nonlinear Static and Dynamic Analysis of Marine Pipelines During Laying, Ship Technology, 38 (1991) 76.

[7] A. Nayfeh, D. Mook, Some Aspects of Modal Interactions in the Response of Beams, in: 28th Structures, Structural Dynamics and Materials Conference, 1987, pp. 777.

[8] C.-M. Chin, A.H. Nayfeh, Three-to-one internal resonances in hinged-clamped beams, Nonlinear Dynamics, 12 (1997) 129-154. 
[9] S.A. Emam, A.H. Nayfeh, Non-linear response of buckled beams to 1: 1 and 3: 1 internal resonances, International Journal of Non-Linear Mechanics, 52 (2013) 12-25.

[10] L. Wang, J. Ma, L. Li, J. Peng, Three-to-one resonant responses of inextensional beams on the elastic foundation, Journal of Vibration and Acoustics, 135 (2013) 011015.

[11] F.B. Seyed, M.H. Patel, Mathematics of flexible risers including pressure and internal flow effects, Marine Structures, 5 (1992) 121-150.

[12] J.H.B. Sampaio Jr, J.R. Hundhausen, A mathematical model and analytical solution for buckling of inclined beam-columns, Applied Mathematical Modelling, 22 (1998) 405-421.

[13] C.E. Mazzilli, C.T. Sanches, O.G.B. Neto, M. Wiercigroch, M. Keber, Non-linear modal analysis for beams subjected to axial loads: Analytical and finite-element solutions, International Journal of Non-Linear Mechanics, 43 (2008) 551-561.

[14] C.E. Mazzilli, C.T. Sanches, Non-linear normal modes of a fixed-moored offshore catenary riser, in: Proc. of XIX Conference AIMETA, 2009.

[15] F. Benedettini, G. Rega, F. Vestroni, Modal coupling in the free nonplanar finite motion of an elastic cable, Meccanica, 21 (1986) 38-46.

[16] N.C. Perkins, Modal interactions in the non-linear response of elastic cables under parametric/external excitation, International Journal of Non-Linear Mechanics, 27 (1992) 233250.

[17] N. Srinil, Analysis and prediction of vortex-induced vibrations of variable-tension vertical risers in linearly sheared currents, Applied Ocean Research, 33 (2011) 41-53.

[18] N. Srinil, H. Zanganeh, A. Day, Two-degree-of-freedom VIV of circular cylinder with variable natural frequency ratio: Experimental and numerical investigations, Ocean Engineering, 73 (2013) 179-194.

[19] I.K. Chatjigeorgiou, S.A. Mavrakos, Nonlinear resonances of parametrically excited risers-numerical and analytic investigation for $\Omega=2 \omega 1$, Computers \& Structures, 83 (2005) 560-573.

[20] Y. Tang, L. Zhu, Y. Li, Nonlinear vibration analysis of combination parametric resonance for TTRs in deep water, Tianjin Daxue Xuebao (Ziran Kexue yu Gongcheng Jishu Ban)/Journal of Tianjin University Science and Technology, 48 (2015) 811-816.

[21] F.K. Alfosail, A.H. Nayfeh, M.I. Younis, Natural frequencies and mode shapes of statically deformed inclined risers, International Journal of Non-Linear Mechanics, 94 (2017) 12-19. [22] S.K. Chakrabarti, R.E. Frampton, Review of riser analysis techniques, Applied Ocean Research, 4 (1982) 73-90.

[23] F.K. Alfosail, A.H. Nayfeh, M.I. Younis, An analytic solution of the static problem of inclined risers conveying fluid, Meccanica, (2016) 1-13.

[24] D. Lucor, H. Mukundan, M.S. Triantafyllou, Riser modal identification in CFD and fullscale experiments, Journal of Fluids and Structures, 22 (2006) 905-917.

[25] A. Placzek, J.F. Sigrist, A. Hamdouni, Numerical simulation of an oscillating cylinder in a cross-flow at low Reynolds number: Forced and free oscillations, Computers and Fluids, 38 (2009) 80-100.

[26] A. Khalak, C.H.K. Williamson, MOTIONS, FORCES AND MODE TRANSITIONS IN VORTEX-INDUCED VIBRATIONS AT LOW MASS-DAMPING, Journal of Fluids and Structures, 13 (1999) 813-851.

[27] M.L. Facchinetti, E. de Langre, F. Biolley, Coupling of structure and wake oscillators in vortex-induced vibrations, Journal of Fluids and Structures, 19 (2004) 123-140. 
[28] O.A. Marzouk, A.H. Nayfeh, New wake models with capability of capturing nonlinear physics, in: ASME 2008 27th International Conference on Offshore Mechanics and Arctic Engineering, American Society of Mechanical Engineers, 2008, pp. 901-912.

[29] M.P. Païdoussis, S.J. Price, E. De Langre, Fluid-structure interactions: cross-flow-induced instabilities, Cambridge University Press, 2010.

[30] A.H. Nayfeh, B. Balachandran, Applied nonlinear dynamics: analytical, computational and experimental methods, John Wiley \& Sons, 2008.

[31] M. Keber, M. Wiercigroch, Dynamics of a vertical riser with weak structural nonlinearity excited by wakes, Journal of Sound and Vibration, 315 (2008) 685-699. 